\title{
Research Article \\ Error Estimates for the Nearly Singular Momentum-Space Bound-State Equations
}

\author{
Yang-Hong Zhang $(\mathbb{D})$ and Jiao-Kai Chen $(1)$ \\ School of Physics and Information Engineering, Shanxi Normal University, Linfen 041004, China \\ Correspondence should be addressed to Jiao-Kai Chen; chenjk@sxnu.edu.cn
}

Received 21 July 2019; Accepted 22 January 2020; Published 1 February 2020

Academic Editor: Zine El Abiddine Fellah

Copyright (c) 2020 Yang-Hong Zhang and Jiao-Kai Chen. This is an open access article distributed under the Creative Commons Attribution License, which permits unrestricted use, distribution, and reproduction in any medium, provided the original work is properly cited.

\begin{abstract}
We present errors of quadrature rules for the nearly singular integrals in the momentum-space bound-state equations and give the critical value of the nearly singular parameter. We give error estimates for the expansion method, the Nyström method, and the spectral method which arise from the near singularities in the momentum-space bound-state equations. We show the relations amongst the near singularities, the odd phenomena in the eigenfunctions, and the unreliability of the numerical solutions.
\end{abstract}

\section{Introduction}

The momentum-space bound-state equations are of great importance, such as the Schrödinger equation, the Dirac equation, the Klein-Gordon equation, the spinless Salpeter equation, the quadratic form of the spinless Salpeter-type equation, the Bethe-Salpeter equation, and so on. Except for the simplest cases, these integral equations should be solved by numerical methods. When employing a numerical method, reliability, the analysis of the error in the computed results, is undoubtedly the foremost consideration and the most important issue. Error estimates and error bounds are related obversely to the reliability of the yielded solutions. Odd phenomena mentioned seldom are related reversely to the reliability, which often appear in unexpected ways. The odd phenomena (In this paper, the considered odd phenomena arise not from the intrinsic properties of the discussed system but from the inappropriate treatment of the momentumspace bound-state equations.) usually connect with the unreliability of the computed results and serve as an indicator of the unreliability, such as Runge's phenomenon $[1,2]$, the Gibbs phenomenon [3,4], the furcation phenomenon in the numerical eigenfunctions [5-7], and the abnormal convergence direction of calculated eigenvalues [8].

Unlike the common singularities which are explicit and known to us, the near singularities [9-11] or numerical sin- gularities [5-8] are prone to being neglected. Although the discussed problems are not singular analytically in the domain, the near singularity will impair the accuracy of the numerical solutions, sometimes even destroys the reliability of the results. We often fall into the illusion that the yielded numerical results are reliable and with expected accuracy. The illusion is reinforced when we find the numerical solutions are stable as we adjust the numerical and physical parameters. The odd phenomena emerging in the calculated results remind us that the numerical results are questionable.

The paper is organized as follows. In Section 2, the discussions on the error estimates for the nearly singular integrals and nearly singular momentum-space bound-state equations are presented. The relations between the near singularities and the reliability of the numerical solutions are discussed. The conclusions are in Section 3.

\section{Near Singularity and Error Estimates}

In this section, we at first present the definition of the near singularity. Then, we give the errors of quadrature rules for the nearly singular integrals. Finally, we discuss errors arising from the near singularities in the momentum-space boundstate equations and the relations amongst the near singularities, the odd phenomena in the numerical eigenfunctions, and the unreliability of the obtained solutions. 
2.1. Near Singularity. Let $f\left(p ; \beta \neq \beta_{s}\right) \in C[a, b]$, where $\beta$ is a parameter and $\beta_{s}$ is the critical value. Let $p_{s}, p \in[a, b]$. If

$$
\lim _{\substack{p \rightarrow p_{s} \\ \beta \rightarrow \beta_{s}}} f(p ; \beta)= \pm \infty
$$

$f(p ; \beta)$ is nearly singular in the neighborhood of $p_{s}$ as $0<$ $|\Delta \beta| \ll 1$ where $\Delta \beta=\beta-\beta_{s}$. $p_{s}$ is the nearly singular point. $\beta$ is the nearly singular parameter. If $\beta=\beta_{s}$, the near singularities become common singularities. The nearly singular function $f(p ; \beta)$ has a near singularity at $p_{s}$ of order $k$ if $k>0$ satisfies

$$
\lim _{p \rightarrow p_{s}} f\left(p ; \beta_{s}\right)\left(p-p_{s}\right)^{k}=c
$$

where $c$ is a nonzero number. The orders of the nearly singular functions are analogous to the orders of poles.

We concentrate on the momentum-space bound-state equations, but the conclusions will be general. Without loss of generality, the radial eigenvalue integral equation can be written as

$$
E \phi(p)=\int K(p, q, \beta) \phi(q) q^{2} d q
$$

where $\beta$ represents all the other parameters. The usual near singularities (or numerical singularities) are logarithmic near singularity in the screened Coulomb potential and the algebraic near singularity in the screened linear potential and the screened Cornell potential [5-8]:

$$
\begin{aligned}
& K(p, q, \beta) \sim \ln \left[(p-q)^{2}\right. \\
& \left.\quad+\beta^{2}\right],\left[(p-q)^{2}+\beta^{2}\right]^{s^{\prime}}, \quad s^{\prime}=-1, \beta_{s}=0 .
\end{aligned}
$$

The numerical solutions of Equation (3) behave badly as $\beta$ which is in the vicinity of $\beta_{s}$ because the kernel $K(p, q$, $\beta$ ) is not well treated in numerical integration which leads to a large error when the numerical method is employed to solve Equation (3).

2.2. Errors of Quadrature Rules. Consider the nearly singular integral:

$$
I(p)=\int_{-1}^{1} K(p, q, \beta) d q
$$

Applying the Newton-Cotes quadrature rules directly to $I(p)$ in which $p$ can be regarded as a constant, we have

$$
Q_{n}(K)=\sum_{i=0}^{n} w_{i} K\left(p, q_{i}\right)
$$

where $h=2 / n, q_{i}=-1+i h$ for $i=0,1, \cdots, n$, and $w_{i}$ are weights.

Although for large $n$ a Newton-Cotes quadrature rule will sometimes suffer from catastrophic Runge's phenomenon, we want to discuss the error of the Newton-Cotes quadrature rules [12]:

$$
R_{n}(f)=I(f)-Q_{n}(f)= \begin{cases}C_{n}^{1} h^{n+2} f^{(n+1)}(\zeta), & \text { for odd } n \\ C_{n}^{2} h^{n+3} f^{(n+2)}(\zeta), & \text { for even } n\end{cases}
$$

where $\zeta \in[a, b], h=(b-a) / n$, and

$$
\begin{aligned}
C_{n}^{1} & =\frac{1}{(n+1) !} \int_{0}^{n} \mu(\mu-1) \cdots(\mu-n) d \mu, \\
C_{n}^{2} & =\frac{1}{(n+2) !} \int_{0}^{n} \mu^{2}(\mu-1) \cdots(\mu-n) d \mu .
\end{aligned}
$$

After calculation, we have

$$
C_{n}^{1} \sim C_{n}^{2} \sim \frac{n}{(n+1) !}\left[\left(\frac{n}{2}\right) !\right]^{2}
$$

Consider the nearly singular kernel which takes the form

$$
K(p, q, \beta)=\frac{1}{(p-q)^{2}+\beta^{2}}
$$

The maximum norm of the derivative of the kernel (10) [13] reads

$$
\left\|K^{(2 n)}\right\|_{\infty}=\left|K^{(2 n)}(q, q)\right|=\frac{(2 n) !}{\beta^{2 n+2}} .
$$

If the nearly singular kernel is logarithmic

$$
K(p, q, \beta)=\ln \left[(p-q)^{2}+\beta^{2}\right]
$$

the maximum norm of the derivative of the kernel (12) reads

$$
\left\|K^{(2 n)}\right\|_{\infty}=\left|K^{(2 n)}(q, q)\right|=\frac{(2 n) !}{n \beta^{2 n}} .
$$

Using Equations (11), (13), and (7), we show that the error arising from the near singularities (Equations (10) and (12)) will dominate the error $R_{n}(K)$ as $\beta$ is small. That is, small $\beta$ results in the unreliability of the numerical results.

Using Equations (9), (11) (or (13)), and Stirling's formula,

$$
n !=\sqrt{2 \pi} n^{n+1 / 2} \exp \left(-n+\frac{\theta}{12 n}\right) \quad(n>0,0<\theta<1)
$$

finite error of Equation (7) demands

$$
\beta \geq \beta_{\mathrm{c}}, \quad \beta_{\mathrm{c}} \approx \frac{1}{e}=0.368,
$$


where $\beta_{c}$ is a critical value. As $\beta>\beta_{c}$, the integral (5) becomes the usual integral while $\beta<\beta_{c}$, the integral (5) is nearly singular.

As employing the composite trapezoidal rule and composite Simpson's rule, the theoretical error of the nearly singular integral (5) reads

$$
\begin{aligned}
R_{2}(f) & =-\frac{h^{2}}{6} f^{(2)}(\zeta), \\
R_{3}(f) & =-\frac{h^{4}}{90} f^{(4)}(\zeta), \\
& \zeta \in[-1,1],
\end{aligned}
$$

respectively. For the algebraic near singularity, using Equations (11) and (16), we have

$$
\begin{aligned}
& \left|R_{2}(K)\right| \sim \frac{h^{2}}{3} \frac{1}{\beta^{4}}, \\
& \left|R_{3}(K)\right| \sim \frac{h^{4}}{45} \frac{12}{\beta^{6}} .
\end{aligned}
$$

For not too small $\beta$, the same accuracy as the common integral can be obtained by decreasing the step size to $h \beta^{2}$ and $h \beta^{3 / 2}$ for the composite trapezoidal rule and composite Simpson's rule, respectively. For the logarithmic near singularity, using Equations (13) and (16), we have

$$
\begin{aligned}
& \left|R_{2}(K)\right| \sim \frac{h^{2}}{3} \frac{1}{\beta^{2}}, \\
& \left|R_{3}(K)\right| \sim \frac{h^{4}}{45} \frac{6}{\beta^{4}} .
\end{aligned}
$$

The same accuracy as the common integral can be obtained by decreasing the step size to $h \beta$ for both the composite trapezoidal rule and composite Simpson's rule as $\beta$ is not too small.

In case of the Gauss-Legendre quadrature rule, the integral (5) can be approximated by

$$
Q_{n}(K)=\sum_{i=1}^{n} w_{i} K\left(p, q_{i}\right)
$$

where the nodes $q_{i}$ are the roots of the $n$th Legendre polynomial $P_{n}(q)$ for $i=1,2, \cdots, n$,

$$
w_{i}=\frac{2}{\left(1-q_{i}^{2}\right)\left[P_{n+1}^{\prime}\left(q_{i}\right)\right]^{2}} .
$$

In the above equation, prime stands for the derivative. The theoretical error formula for the Gauss-Legendre rule on the interval $[-1,1]$ reads [14].

$$
\left|R_{n}(f)\right| \leq \frac{2^{2 n+1}(n !)^{4}}{(2 n+1)[(2 n) !]^{3}}\left\|f^{(2 n)}\right\|_{\infty} .
$$

Using Equations (11) and (14), Equation (21) reduces to

$$
\left|R_{n}(K)\right| \leq \frac{2 \pi}{(2 \beta)^{2 n+1}} \exp \left(\frac{\theta}{4 n}\right) .
$$

Evidently, finite error demands

$$
\beta \geq \beta_{\mathrm{c}}, \quad \beta_{\mathrm{c}}=\frac{1}{2},
$$

as $n \longrightarrow \infty$. The same result can be obtained for the logarithmic near singularity. Equation (23) is different from the asymptotic error estimate for $\beta \ll 1$ given through the contour integral [15]:

$$
\left|R_{n}(K)\right| \lesssim \frac{2 \pi}{\beta} \exp (-2 \beta n), \quad n \longrightarrow \infty .
$$

In practice, small $\beta$ will impair the accuracy as $n$ is finite.

2.3. Errors Arising from the Near Singularities in the Momentum-Space Bound-State Equations. In case of the approximation method, we can have the matrix equation from Equation (3):

$$
E^{N} M \mathbf{a}=L \mathbf{a}, \quad E^{N} \equiv \frac{\mathbf{a}^{\mathrm{T}} L \mathbf{a}}{\mathbf{a}^{\mathbf{T}} M \mathbf{a}},
$$

where

$$
\psi(p) \approx \psi^{N}(p)=\sum_{i=1}^{N} a_{i} h_{i}(p)
$$

$\mathbf{a}=\left\{a_{i}, \cdots, a_{N}\right\}$, and

$$
\begin{aligned}
L_{i j} & =\int h_{i}^{\dagger}(p) K\left(p, p^{\prime}\right) h_{j}\left(p^{\prime}\right) p^{2} p^{\prime 2} d p d p^{\prime}, \\
M_{i j} & =\int h_{i}^{\dagger}(p) h_{j}(p) p^{2} d p .
\end{aligned}
$$

If the set $\left\{h_{i}\right\}$ is orthonormal, $M$ becomes the identity. In the Nyström method case, we have from Equation (3)

$$
F\left(\psi^{N}\right)=\frac{\left(\psi^{N}, K \psi^{N}\right)}{\left(\psi^{N}, \psi^{N}\right)}=\frac{\sum_{i, j=1}^{N} c_{i} c_{j} \psi_{i}^{N} K_{i j} \psi_{j}^{N}}{\sum_{i=1}^{N} c_{i} \psi_{i}^{N} \psi_{i}^{N}}
$$

where $\psi_{i}^{N}=\psi^{N}\left(p_{i}\right), K_{i j}=K\left(p_{i}, p_{j}\right), c_{i}$ is the weight for the chosen quadrature rule multiplied by $p_{i}^{2}$. Setting

$$
\frac{\partial F}{\partial \psi_{m}^{N}}=0, \quad m=1,2, \cdots, N,
$$


we can obtain

$$
\frac{\psi_{m}^{N}\left(\sum_{i, j=1}^{N} c_{i} c_{j} \psi_{i}^{N} K_{i j} \psi_{j}^{N}\right)-\left(\sum_{i=1}^{N} c_{i} \psi_{i}^{N} K_{m i}\right)\left(\sum_{i=1}^{N} c_{i} \psi_{i}^{N} \psi_{i}^{N}\right)}{\left(\sum_{i=1}^{N} c_{i} \psi_{i}^{N} \psi_{i}^{N}\right)}=0,
$$

if the matrix $K_{i j}$ is symmetric; that is,

$$
E^{N} \psi_{m}^{N}=\sum_{i=1}^{N} c_{i} K_{m i} \psi_{i}^{N}
$$

The above equation is just the matrix equation yielded by discretizing the integral Equation (3). For the spectral method, the matrix equation obtained from Equation (28) can be written also in the form of Equation (31).

In Equations (25) and (31), the common errors arising from the numerical methods are not given. We concentrate on the errors resulting from the near singularities. For simplicity, Equations (25) and (31) are rewritten as

$$
\varepsilon_{n} \phi_{n i}=\sum_{j=1}^{N} K_{i j} \phi_{n j}
$$

When the near singularities are treated and then the eigenvalue integral Equation (3) becomes less nearly singular or free of near singularities [5-8, 16-18], the obtained matrix equation corresponding to Equation (32) reads

$$
\varepsilon_{n}^{\prime} \phi_{n i}^{\prime}=\sum_{j=1}^{N} K_{i j}^{\prime} \phi_{n j}^{\prime}, \quad K_{i j}^{\prime}=K_{i j}-M_{i j},
$$

where $M_{i j}$ are the additional errors arising from the near singularities. In this section, $\phi_{n i}$ and $\phi_{n i}^{\prime}$ are the unnormalized eigenfunctions belonging to the $n$th eigenvalues $\varepsilon_{n}$ and $\varepsilon_{n}^{\prime}$, respectively. We can assume that the solutions, $\phi_{n}^{\prime}$ and $\varepsilon_{n}^{\prime}$, to (33) are well behaved and are good approximations of the actual solutions while the solutions, $\phi_{n}$ and $\varepsilon_{n}$, to the nearly singular Equation (32) are an approximation to $\phi_{n}^{\prime}$ and $\varepsilon_{n}^{\prime}$ and may be be bad with low accuracy. If Equation (3) is free of near singularities, $M_{i j}=0, \varepsilon_{n}=\varepsilon_{n}^{\prime}$, and $\phi_{n i}=\phi_{n i}^{\prime}$.

Let $i=n, \phi_{n n}^{\prime}=1$, and we have from Equation $(33)[7,19]$

$$
\varepsilon_{n}^{\prime}=\sum_{j=1}^{N} K_{n j}^{\prime} \phi_{n j}^{\prime}
$$

Let $i \neq n$; then, we obtain components of the $n$th eigenfunction:

$$
\begin{aligned}
\phi_{n n}^{\prime} & =1, \\
\phi_{n i}^{\prime} & =\frac{\sum_{j \neq i} K_{i j}^{\prime} \phi_{n j}^{\prime}}{\varepsilon_{n}^{\prime}-K_{i i}^{\prime}} .
\end{aligned}
$$

The same procedure applied to Equation (32) yields the results:

$$
\begin{aligned}
\varepsilon_{n}= & \varepsilon_{n}^{\prime}+\sum_{j=1}^{N} K_{n j}^{\prime} \Delta_{n j} \\
& +\sum_{j=1}^{N} M_{n j}\left(\Delta_{n j}+\phi_{n j}^{\prime}\right), \quad \Delta_{n j}=\phi_{n j}-\phi_{n j}^{\prime},
\end{aligned}
$$

$$
\begin{aligned}
& \phi_{n n}=1, \\
& \phi_{n i}=\frac{\varepsilon_{n}^{\prime} \phi_{n i}^{\prime}+\sum_{j=1}^{N} K_{i j}^{\prime} \Delta_{n j}+\sum_{i \neq j} M_{i j} \phi_{n j}}{\varepsilon_{n}-M_{i i}},
\end{aligned}
$$

where we have used Equation (33). Equations (34)-(37) are exact results. As the Nyström method is employed, $M_{i j}$ will be diagonal. If the elements of $M$ are small, we have from Equations (36) and (37)

$$
\begin{aligned}
& \varepsilon_{n}=\varepsilon_{n}^{\prime}+M_{n n}+\sum_{j=1}^{N} K_{n j}^{\prime} \Delta_{n j}, \\
& \phi_{n n}=1, \\
& \phi_{n i}=\frac{\varepsilon_{n}^{\prime}+\sum_{j=1}^{N} K_{i j}^{\prime} \Delta_{n j} / \phi_{n i}^{\prime}}{\varepsilon_{n}-M_{i i}} \phi_{n i}^{\prime} .
\end{aligned}
$$

From Equations (32)-(39), we can see that $M_{i j}$ are related directly with the near singularities in the integral equation and they result in errors in the eigenvalues and the corresponding eigenfunctions. $M_{n n}$ or $M_{n n} / E_{n}$ can be used as crude error estimate and a sign whether the original Equation (3) can be solved straightforwardly by numerical method. If $M_{n n}$ are large, Equation (3) should be handled to weaken or remove the near singularities in it.

Formulas (37) and (39) can be applied to explain why the furcation phenomenon emerges in the obtained eigenfunctions [5-7]. The alternate wobble of the weights leads to the alternate wobble of $M_{i i}$ which results in the wobble of the point of the yielded eigenfunction - that is, the furcation with branches emerges. Equations (36) and (38) give the relation between the furcation phenomenon and error resulting from the near singularities in the obtained eigenvalues. As the furcation phenomenon becomes stronger, $M_{i i}$ will be larger and the integrand is more nearly singular; then, the errors will increase. The furcation phenomenon in the numerical eigenfunctions indicates not only the unreliability of the numerical results but also the bad behavior of the integral Equation (3).

\section{Conclusions}

We present errors of quadrature rules for the nearly singular integrals in the momentum-space bound-state equations. Then, the critical value of the nearly singular parameter is given. We give error estimates for the expansion method, the Nyström method, and the spectral method which arise from the near singularities in the momentum-space boundstate equations. We show that the relations amongst the near 
singularities, the odd phenomena in the eigenfunctions, and the unreliability of the numerical solutions.

\section{Data Availability}

The data used to support the findings of this study are included within the article.

\section{Conflicts of Interest}

The authors declare that they have no conflicts of interest.

\section{Acknowledgments}

The authors are supported by the Shanxi Provincial Natural Science Foundation under Grant No. 201901D111289.

\section{References}

[1] C. Runge, "Über empirische Funktionen und die Interpolation zwischen äquidistanten Ordinaten," Zeitschrift für Mathematik und Physik, vol. 46, p. 224, 1901.

[2] E. W. Cheney, Introduction to Approximation Theory, McGraw-Hill, New York, 1966.

[3] J. W. Gibbs, "Fourier's series," Nature, vol. 59, p. 606, 1899.

[4] E. Hewitt and R. E. Hewitt, "The Gibbs-Wilbraham phenomenon: an episode in Fourier analysis," Archive for History of Exact Sciences, vol. 21, no. 2, pp. 129-160, 1979.

[5] J. K. Chen, "Extended Simpson's rule for the screened Cornell potential in momentum space," Physical Review D, vol. 86, no. 3 , article 036013, 2012.

[6] J.-K. Chen, "Erratum: Extended Simpson's rule for the screened Cornell potential in momentum space [Phys. Rev. D 86, 036013 (2012)]," Physical Review D, vol. 89, no. 9, article 099904, 2014.

[7] J. K. Chen, "Nyström method for the coulomb and screened coulomb potentials," Few-Body Systems, vol. 54, no. 11, pp. 2081-2095, 2013.

[8] J. K. Chen, "Spectral method for the Cornell and screened Cornell potentials in momentum space," Physical Review D, vol. 88, no. 7, article 076006, 2013.

[9] G. Monegato and A. P. Orsi, "Product formulas for Fredholm integral equations with rational kernel functions," in Numerical Integration III. International Series of Numerical Mathematics / Internationale Schriftenreihe zur Numerischen Mathematik / Série internationale d'Analyse numérique, $\mathrm{H}$. Braß and G. Hämmerlin, Eds., vol. 85, p. 140, Birkhäuser, Basel, 1988.

[10] P. Pastore, “The numerical treatment of Love's integral equation having very small parameter," Journal of Computational and Applied Mathematics, vol. 236, no. 6, pp. 1267-1281, 2011.

[11] K. E. Atkinson and L. F. Shampine, "Algorithm 876," ACM Transactions on Mathematical Software, vol. 34, no. 4, pp. 120, 2008

[12] K. E. Atkinson, An Itroduction to Numerical Analysis, John Wiley \& Sons, Canada, 1988.

[13] H. Brass and K. Petras, Quadrature Theory, Volume 178 of Mathematical Surveys and Monographs, American Mathematical Society, Providence, Rhode Island, 2011.
[14] M. Abramowitz and I. A. Stegun, Handbook of Mathematical Functions, U.S. Govt. Print. Off, Washington, 10th edition, 1972.

[15] L. Klinteberg and A. K. Tornberg, "Error estimation for quadrature by expansion in layer potential evaluation," Advances in Computational Mathematics, vol. 43, no. 1, pp. 195-234, 2017.

[16] J. K. Chen, "Numerical solutions of one reduced Bethe-Salpeter equation for the coulombic bound states composed of virtual constituents," Journal of the Physical Society of Japan, vol. 87, no. 4, article 044101, 2018.

[17] J. K. Chen, "Numerical solutions of the Maung-NorburyKahana equation with the coulomb potential in momentum space," Revista mexicana de física, vol. 64, no. 1, p. 8, 2018.

[18] J. K. Chen, "Numerical solutions of the quadratic form of the spinless Salpeter-type equation," Romanian Journal of Physics, vol. 62, p. 119, 2017.

[19] R. Courant and D. Hilbert, Methods of Mathematical Physics I, Interscience, New York, 1966. 